\title{
Video Monitoring of Pulverized Coal Injection in the Blast Furnace
}

\author{
Wolfgang Birk ${ }^{\dagger}$, Olov Marklund ${ }^{\star \dagger}$, and Alexander Medvedev ${ }^{\dagger}$ \\ ${ }^{\star}$ Corresponding author: Tel. +46 920 91064, Fax +46 920 72082, eMail Olov.Marklund@ sm.luth.se \\ ${ }^{\dagger}$ Department of Comp. Science and Electr. Eng., Luleå University of Technology, SE-971 87 Luleå, Sweden
}

\begin{abstract}
A novel approach for monitoring and control of the coal powder injection in a blast furnace is presented and discussed. Image analysis of video recordings is used as a means to estimate the instantaneous coal flow. Initial experiments at the blast furnace no 3 of SSAB Tunnplåt AB Luleå, Sweden, have been performed and first hand results on modelling and control of a single injection line are given.
\end{abstract}

\section{INTRODUCTION}

In the blast furnace process, coke is usually used as fuel and reduction agent. Since coal is $40 \%$ cheaper than coke, injecting pulverized coal instead of using coke is economically beneficial. According to [1], the share of pulverized coal compared with coke as fuel will rise from $36 \%$ to $50 \%$ by the year 2015 .

A coal injection plant is a highly automated plant, where incoming raw coal is stored, ground, dried and finally injected into the blast furnace. During operation, human interaction is only needed for making set-point adjustments. Fig. 1 shows the structure of the plant, where only the injection vessels, distributor, the blast furnace and the monitoring equipment are depicted. The control of the injection process is complicated due to the two phase nature of the injected flow (gas plus particles). Direct measurement of the coal mass flow is difficult since a flow meter installed on the injection pipe only provides a measurement of the cumulative flow of gas and coal powder where coal content might significantly vary.

The total coal flow leaving the injection plant is distributed into several parallel lines. In [2] it is shown how the total coal flow can be stabilized. The problem at hand is to achieve a desired injection distribution of the flow around the blast furnace. Therefore, estimation and control of the coal flow in each line is required.

The coal mass flow estimation is derived from images of the coal jet entering the raceway of the blast furnace. For each injection line, images are acquired in real time using a video camera monitoring the scene through a peephole in the tuyere (see Fig. 1). With the aid of an image analysis scheme, an estimate of the instant coal mass flow is then obtained.

As the coal mass flow to the distributor can be assumed to be constant [3], the flow has to be distributed according to a desired profile between a number of injection lines. Each of these lines is equipped with a flow control valve. Using the estimated coal mass flow as the measurement output and the valve as actuator a mathematical model of the injection process is identified from process data. The obtained model is then used to design a controller, which stabilizes the coal mass flow in the injection line.

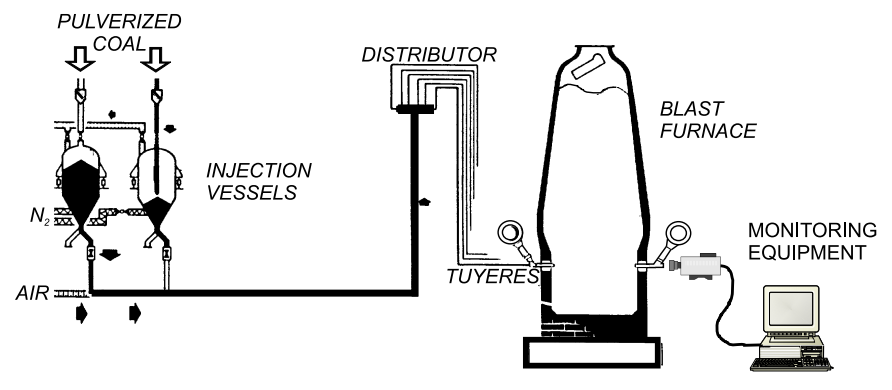

Fig. 1. Setup for video monitoring. Parts of the coal injection plant, distributor, blast furnace and monitoring equipment

\section{FLOW ESTIMATION}

A representative scene of the coal injection process, as recorded with the video camera, is depicted in Fig. 2a. A digitized video frame will below be denoted $F(i, j, \tau)$ where $(i, j)$ represent the pixel coordinates and $\tau$ is a discrete time parameter (the shorter notation $F(\tau)$ will henceforth be used).

Initially a binary mask $R$, representing the actual region of interest, is produced. This is done by forming an image $D=$ $\sum|F(\tau)-F(\tau-1)|, \tau=2,3, \ldots$, i.e. an image consisting of accumulated differences (due to motion) between subsequent video frames during a sufficiently long period of time (typically a few minutes).

Since the furnace wall and the tuyere will make up a static scene, no differences will be detected in those regions of the frames. The coal plume as well as the gaseous interior of the furnace, on the other hand, will display a highly dynamic behavior resulting in detectable differences. Masks such as the one depicted in Fig. $3 \mathrm{~b}$ can thus be straightforwardly produced from a final difference image $D$ by simply selecting the region consisting of values that are high enough (indicating motion).

Once a mask $R$ has been created, the region of interest in all subsequent frames $F(\tau)$ can be conveniently detected and the actual measuring procedure (i.e. detecting and analyzing the actual plume inside the region of interest) can be started.

First a grayscale histogram [4] for the set of pixels $R_{\tau}$, the pixels in a frame $F(\tau)$ that are covered by the mask $R$, is formed and analyzed. The histograms are typically of a bimodal nature with the darker pixel values (representing the plume) and the brighter pixel values (representing the gaseous background) forming two well separated 'hills'. This enables the detection of an optimal threshold value $T(\tau)$ such that a subset $P_{\tau}$, representing the coordinates of pixels making up the actual plume, 


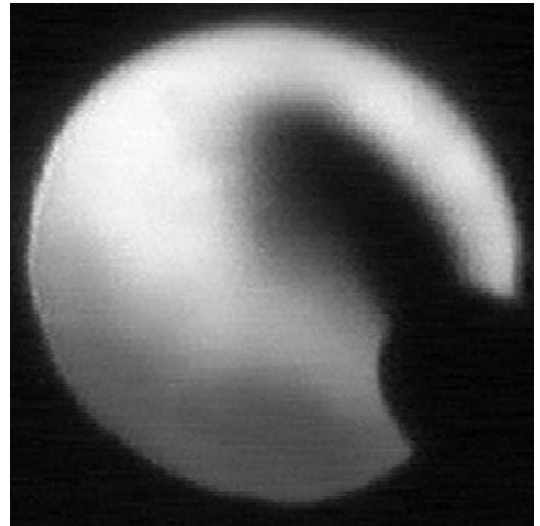

(a)

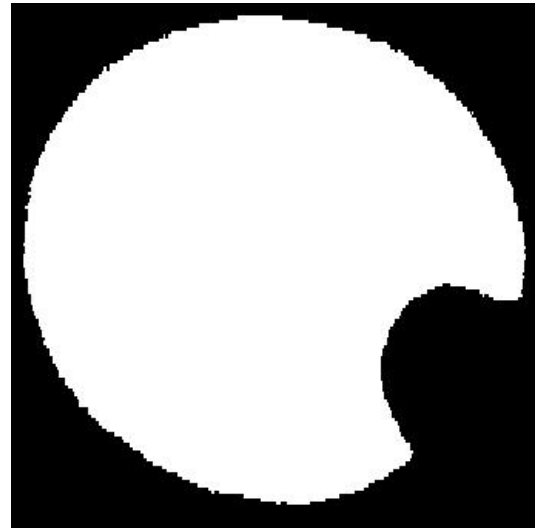

(b)

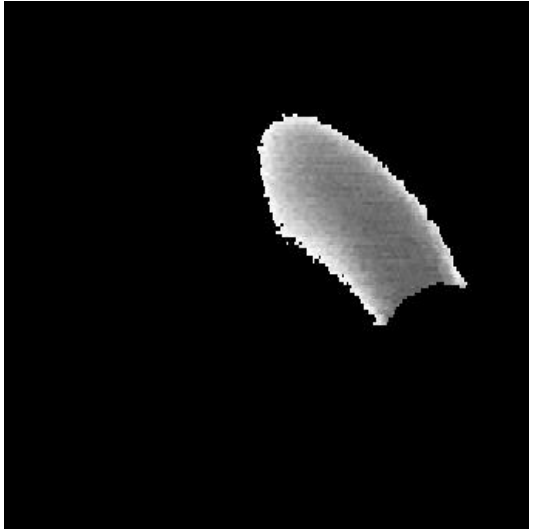

(c)

Fig. 2. (a) Digital image depicting the coal injection process. (b) The binary mask R. (c) The projected plume (segmented from the rest of the image).

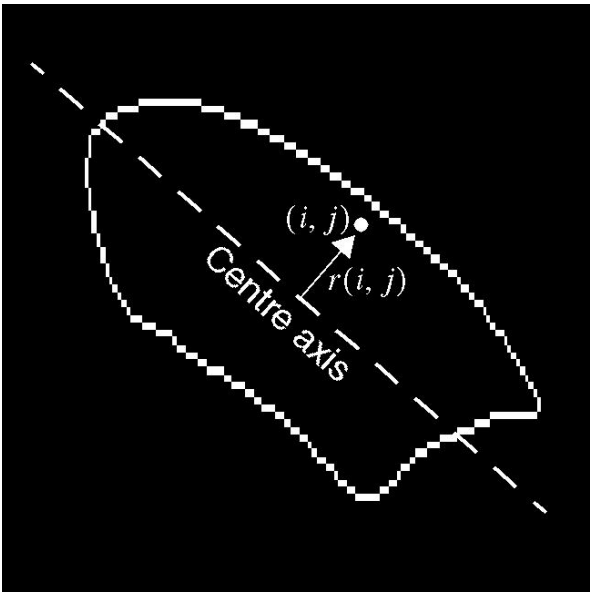

Fig. 3. Elementary volume transform

can be determined through the decision rule: $(i, j) \in P_{\tau}$ if $(i, j) \in R_{\tau}$ and $F(i, j, \tau)<T(\tau)$.

The resulting set $P_{\tau}$ is then further processed using a chain of binary morphology operations [4] in order to produce a simple connected area inside $R$ representing a 2-D projection of the plume.

The final processed set $P_{\tau}$ of image coordinates (representing the projected plume) is then used to form a relative estimate $m(\tau)$ of the injected coal mass at time $\tau$ through the operation

$$
m(\tau)=\sum_{(i, j) \in P_{\tau}} f\left(R_{\tau}, i, j, \tau\right)
$$

where the optimal functional form of $f(\cdot)$ is still an open research issue. The most straightforward choice is $f(\cdot)=A$, where $A$ is a constant, i.e. a value directly proportional to the total area covered by the pixels in $P_{\tau}$ is used as relative estimate.

A more sophisticated choice is

$$
f(\cdot)=f_{\alpha}(\cdot)=A_{\alpha}[R(i, j, \tau)+\epsilon]^{-\alpha},
$$

where $A_{\alpha}, \alpha$, and $\epsilon$ are constants greater than zero ( $\epsilon$ is included to prevent division by zero). The rationale behind this choice is to give coordinates in $P_{\tau}$ representing low grayscale values a higher weight (due to the exponent $-\alpha$ ) based on the assumption that they represents regions with higher coal powder density.

As a first attempt for a 3-D model, a rotational symmetric plume has been assumed. The transformation from projected 2$\mathrm{D}$ data to volume data is carried out as follows. First the equation for the center axis of the plume (see Fig. 3) is estimated through principal axis analysis on $P_{\tau}$, and then the weighted functional

$$
f(\cdot)=2 \pi r(i, j) f_{\alpha}(\cdot),
$$

where $r(i, j)$ is the orthogonal distance from the center axis to the position $(i, j)$ (as depicted in Fig. 3), is applied in (1). Using the accumulated flow over a time period of several minutes the estimate $m(\tau)$ can be calibrated (i.e. a suitable value for the contant $A_{\alpha}$ is determined).

A comparison between one such estimate and a corresponding measurement using a Coriolis flow meter (measuring the cumulative gas/coal flow) is shown in Fig. 4. Obviously the two measurements exhibit a very similar dynamic behavior.

The use of more complex 3-D models, determined by matching parameters (extracted through image analysis of the video feed) with approximate solutions of the governing equations describing two phase flows in systems such as the here discussed, is currently under investigation.

\section{SINGLE LINE FLOW CONTROL}

The two-phase flow that is leaving the coal injection plant is usually conveyed over several hundred meters until it reaches the coal distributor. Even if the coal flow is perfectly stabilized directly after the injection plant, fluctuations appear in the flow as the coal particles are conveyed in a so-called dense flow [5].

Distribution of pulverized coal over the blast furnace diameter is provided by the fixed construction of the distributor. No actuators are used to modify the achieved flow distribution pro- 
(a)

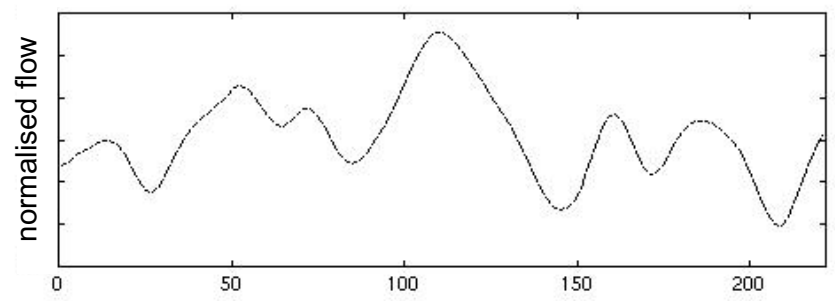

(b)

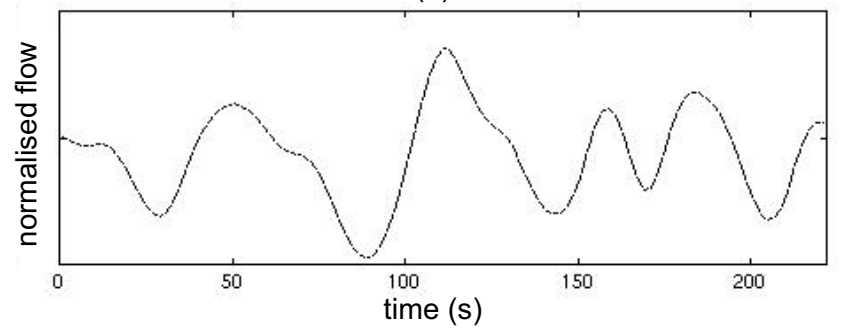

Fig. 4. Comparison of normalised estimated flows. (a) Using an image sequence. (b) Using a Coriolis flow meter.

file. Thus, any fluctuations or other disturbances can not the attenuated by this open-loop structure.

Introducing single line flow control, see Fig. 5, where the measured flow in a single line $q_{C i}$ is fed back to the actuator $u_{C i}$ via a controller, can remove induced fluctuations and attenuate disturbances. Moreover, tracking can be introduced.

Furthermore, it enables the plant operators to achieve desired flow profiles around the blast furnace diameter. Clearly, the energy supply to the blast furnace can be directed to certain regions in the furnace and in turn, temperature control might become possible.

The desired flow distribution profile can be set in two different ways:

1. The sum of the flows in all tuyeres is set and the flow in each tuyere is a ratio of the sum. Thereby, it is ensured that provided flow from the PCI plant can be injected.

2. The flow in each tuyere is set as an absolute value. Thus, the provided flow from the PCI plant has to be adjusted to the sum of the individual flows.

\section{A. Implications}

Beside the potential of single line flow control, the application has implications for the operation of PCI plants. Mainly the flow control of the plant has to be tightened and the transport delay between the injection vessels and the coal distributor has to be considered.

Variations in the provided coal flow from the PCI plant will directly propagate to each single line and cause counteraction by the controllers. As each control loop is dynamically coupled via the coal distributor it has to be investigated how this multivariable control problem can be successfully solved.

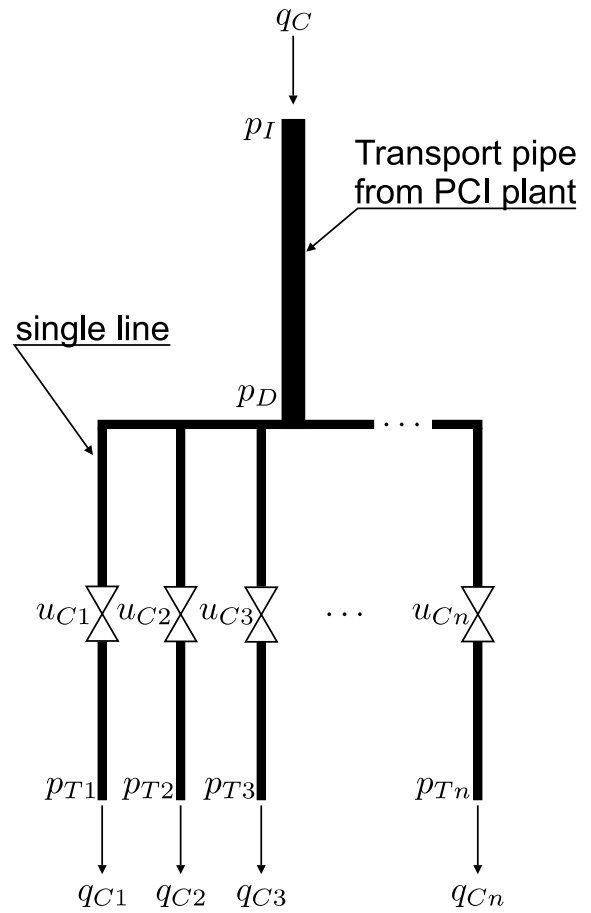

Fig. 5. Physical structure of a single line control setup

\section{B. Video feedback control}

There are two ways to measure the coal flow in an injection line, by using a flow meter, e.g. a Coriolis flow meter, or by extracting flow information from a video image of the coal jet entering the tuyere.

Considering the uncertainties of the flow measurement and the costs involved with the purchase of the flow meters, video image analysis is an economically attractive alternative. Moreover, the video image is a rich information source, where more than only flow information can be found.

From a maintenance point of view, video observation gives the operators another degree of freedom, as maintenance of tuyeres can be based on the evaluation of the video image of the tuyeres.

Here, the flow estimation algorithm is used as a soft-sensor that provides a measurement of $q_{C i}$. It is important to note, that the soft-sensor has to be calibrated properly, as the performance of the closed loop system depends on the quality of the measurement.

Since the soft-sensor is part of the dynamic model for the single line, changes in the zoom or position of the scene in the image frame affect model gains or, even worse, model dynamics.

\section{Actuator}

Using the previously described flow estimation algorithm, a flow measurement is extracted from the video image and fed back. In order to affect the flow in the single line the valve in Fig. 6 is used. 


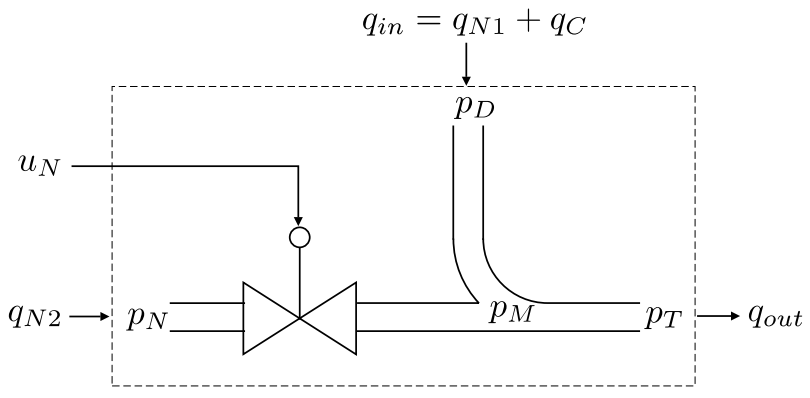

Fig. 6. Schematic drawing of the flow control valve

It can be seen that the single line merges with a gas pipe, which can introduce a gas flow into the single line via a valve. Opening the valve causes a flow $q_{N 2}$ to the merge. As the total mass flow out of the actuator can not increase, the in-flow $q_{\text {in }}$ has to decrease. Thus, the coal flow is reduced. Clearly, the actuator has a negative gain and closing the valve increases the coal flow.

\section{PHYSICAL MODEL}

A physical model for the dynamical behavior of the coal flow in the injection line from the distributor to the observed coal plume in the tuyere consists of three parts.

1. Dynamics of the actuator.

2. Transport delay of from the actuator to the tuyere.

3. Coal plume dynamics.

The dynamics of the actuator is based on the simplification that the flow dynamics in the pipe can be neglected and that the coal particle flow can be governed by fluid flow equations.

Furthermore, it is assumed that the mass flow balance at the actuator output is constant. It can be constituted that three mass flows are entering the merging point

$$
\begin{aligned}
\dot{m}_{N 1} & =q_{N 1} \frac{p_{D}}{R T_{D}} \\
\dot{m}_{N 2} & =q_{N 2} \frac{p_{M}}{R T_{M}} \\
\dot{m}_{C} & =q_{C} \rho_{C},
\end{aligned}
$$

where $\dot{m}_{\bullet}$ and $q_{\bullet}$ denote mass flow and volume flow, respectively. Assuming, distributor and merging point are close to each other, the pressure $p_{D} \approx p_{M}$ and $T_{D} \approx T_{M}$.

Thus, the mass balance is obtained

$$
\begin{aligned}
\dot{m}_{\text {out }} & =\dot{m}_{C}+\dot{m}_{N 1}+\dot{m}_{N 2} \\
& =q_{C} \rho_{C}+q_{N 1} \frac{p_{M}}{T_{M}}+q_{N 2} \frac{p_{M}}{T_{M}} \\
& =\text { const }
\end{aligned}
$$

Clearly, an increased flow $q_{N 2}$ yields a reduced flow $q_{N 1}+q_{C}$.

In (2) the flow $q_{N 2}$ is given as a flow through an 'equal percentage' valve [6]. It is given by

$$
q_{N 2}=k_{N 2} f_{g a s}\left(p_{N}, p_{M}\right) g_{e x p}\left(u_{N}\right)
$$

where $k_{N 2}$ is a factor that maps the opening characteristics $g_{\exp }$ to an area. The function $f_{\text {gas }}$ describes the flow through a stricture [7].

From the merging point in the actuator to the end of the injection line in the tuyere there is a transport delay $t_{d}$. Furthermore, it is assumed that the entering coal flow propagates through the pipe unaltered. Hence, the dynamics can be given in the Laplace domain as

$$
G_{d}(s)=e^{-t_{d} s}
$$

The dynamics of the coal plume are assumed to be fast and reach steady state instantaneously. Moreover, when the twophase flow enters the tuyere as a jet, the two phases are seen separately due to the optical properties of nitrogen. Assuming, that the coal particles remain visible, which means unburned, for a constant time, the amount of visible particles characterizes the flow. Therefore, the particle number $N_{C}$ is directly proportional to the coal flow in the injection line

$$
N_{C}=k_{C} q_{C}
$$

Combining (2), (3) and (4) a complete physical model is found.

\section{MOdel PARAMETERS}

Several contiguities in the physical model are unknown and have to be determined from experiments at the blast furnace.

To this end, the actuator has to be affected and the coal flow has to be measured simultaneously by the soft sensor. The setup depicted in Fig. 1 is modified so that the computer is connected to the actuator via a buffer amplifier.

The following experiments are performed:

- Static experiment:

The valve is opened from closed to fully open in a number of equidistant steps. Each step is held for around 60 seconds. It is assumed that steady state is reached after 30 seconds.

- Dynamic experiments:

A pseudo random noise signal is generated and send to the actuator. Amplitude, offset and frequency range are varied.

Evaluation of the experiments show that the coal plume in the blast furnace is not only affected by the flow in the injection pipe but also dynamically affected by the blast air flow in the tuyere. During the design of the soft sensor, the effect of this transversal flow is assumed static and thus, it is compensated by a static gain.

Fig. 7 shows logged data of the input signal to the actuator and the output signals from the soft sensor. Correlation analysis shows a weak correlation (less than 20\%) between the actuator signal and flow measurement, Fig. 8. Consequently, the dynamic effects of the blast air flow have to be considered in the flow measurement and the soft sensor has to be re-designed.

It can be concluded that more experiments have to be performed in order to get a better understanding on the behavior of the coal particle jet in the blast air flow. 

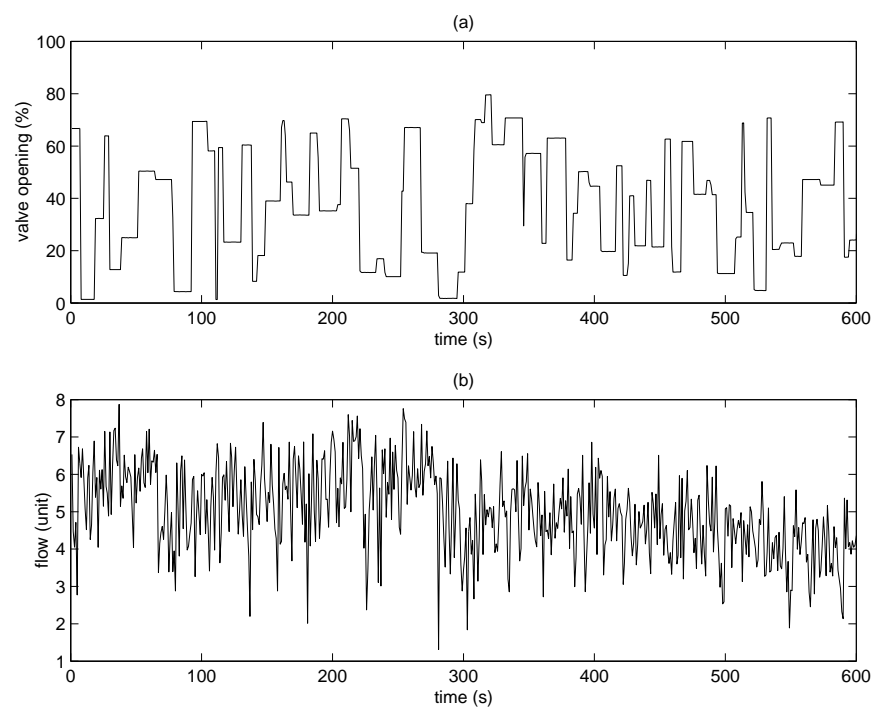

Fig. 7. Process data. (a) Pseudo random noise input signal. (b) Estimated flow from video image.

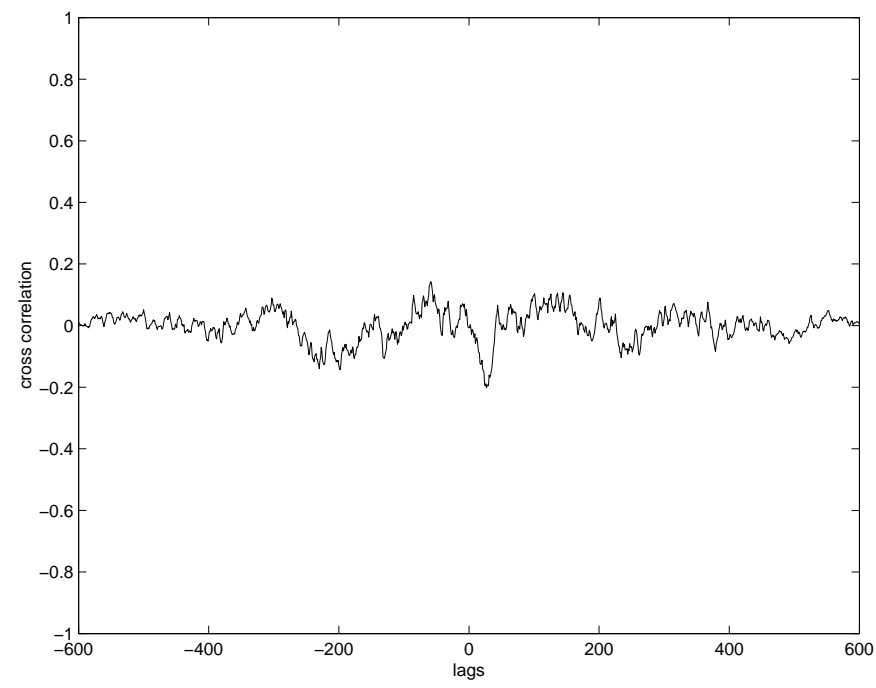

Fig. 8. Correlation between input and output signals in units of the normalizes autocorrelation of the signals

\section{CONCLUSIONS AND FURTHER WORK}

A novel method for estimating and controlling the pulverized coal flow in a single injection line to the blast furnace has been presented.

The design of a soft sensor for coal flow estimation in a blast furnace tuyere is discussed and applied to logged data. The results indicate that reliable coal flow estimation from video images is viable and can be used as a soft sensor for control purposes.

Using the soft sensor output, control of the coal flow in a single injection line becomes applicable. Implications for plant operation are pointed out.

A physical model for the dynamical behavior from the actuator to the observed coal plume has been derived. Initial experi- ments have shown that the assumption on the blast air flow does not hold. The effect of the blast air on the coal plume dynamics has to be studied more thoroughly.

More experiments have to be performed in order to get a better understanding of the model dynamics. Utilizing experimental data, model parameters have to be derived and the model validation has to be carried out. Subsequently, a model-based controller can be designed for the single line.

\section{ACKNOWLEDGEMENT}

The authors want to thank SSAB Tunnplåt $A B$ in Luleå, Sweden for making the plant and maintenance personnel available. A special thank to Juan Arellano Sanzol who helped with the experiments at the plant. Financial support from the Swedish National Board for Industrial and Technical Development (NUTEK) is also gratefully acknowledged.

\section{REFERENCES}

[1] American Iron and Steel Institute, "Steel industry technology roadmap." At http://www.steel.org/MandT/contents.htm, February 1998.

[2] W. Birk and A. Medvedev, "Pressure and flow control of a pulverized coal injection vessel," IEEE Transactions on Control Systems Technology, vol. 8, pp. 919-929, November 2000.

[3] W. Birk, A. Johansson, and A. Medvedev, "Model-based control for a fine coal injection plant," IEEE Control Systems Magazine, vol. 19, pp. 33-43, February 1999.

[4] A. Jain, Fundamentals of Digital Image Processing. Prentice-Hall, Englewood Cliffs, N.J., 1989.

[5] G. Klinzing, R. Marcus, F. Rizk, and L. Leung, Pneumatic conveying solids - A theoretical and practical approach. Chapman and Hall, cop., London, 1997.

[6] Control valve handbook. Fischer Controls, 1977.

[7] A. Johansson and A. Medvedev, "Model based leakage detection in a pulverized coal injection vessel," IEEE Transactions on Control Systems Technology, vol. 7, pp. 675-682, November 1999. 